\title{
Integers free of prime divisors from an interval, I
}

\author{
by \\ Andreas Weingartner (Cedar City, UT)
}

1. Introduction. Let $\Gamma(x, y, z)$ be the number of positive integers not exceeding $x$ which are free of prime divisors from the interval $(z, y]$. Our interest in this function is two-fold. On the one hand it constitutes a generalization of the functions

$$
\Psi(x, z)=\sum_{\substack{n \leq x \\ P^{+}(n) \leq z}} 1 \text { and } \Phi(x, y)=\sum_{\substack{n \leq x \\ P^{-}(n)>y}} 1,
$$

where $P^{+}(n)$ and $P^{-}(n)$ denote the largest and smallest prime divisor of $n$, respectively. These functions play an important role in various applications of number theory, such as an improvement of the Selberg sieve by Jurkat and Richert [7], a study of the distribution of $k$ th power residues of primes by Davenport and Erdős [5] and a general convolution method by Daboussi [4]. The behaviour of $\Psi$ and $\Phi$ has been studied intensively by several authors (see, for example, $[1,2]$ ). For an overview on results on $\Psi$ and $\Phi$ see Tenenbaum [14].

On the other hand, the behaviour of $\Gamma(x, y, z)$ is worth studying in its own right. The quantity $\Gamma(x, y, z)$ arises in applications such as the construction of large prime gaps (see Rankin $[8,9]$ or Schönhage [13]).

Throughout we will use the notation

$$
u=\frac{\log x}{\log y} \quad \text { and } \quad v=\frac{\log x}{\log z} .
$$

Dickman's function @ is defined to be the unique continuous solution to the difference-differential equation

$$
v \varrho^{\prime}(v)+\varrho(v-1)=0 \quad(v>1),
$$

2000 Mathematics Subject Classification: Primary 11N25. 
together with the initial condition

$$
\varrho(v)=1 \quad(0 \leq v \leq 1) .
$$

Let $\varrho(v)=0$ for $v<0$ and extend $\varrho^{\prime}(v)$ to $v=1$ by right continuity.

For $u>1$, the Buchstab function $\omega(u)$ is defined as the unique continuous solution to the difference-differential equation

$$
(u \omega(u))^{\prime}=\omega(u-1) \quad(u>2)
$$

with initial condition

$$
u \omega(u)=1 \quad(1 \leq u \leq 2) .
$$

Let $\omega(u)=0$ for $u<1$. Define $\omega$ at 1 and $\omega^{\prime}$ at 1 and 2 by right continuity.

The functions $\varrho(v)$ and $\omega(u)$ arise in well known estimates for the functions $\Psi(x, z)$ and $\Phi(x, y)$. We will make use of the following two results. The first estimate is an easy consequence of a well known estimate of de Bruijn [2] (for a slightly weaker estimate see Theorem III.5.6 in Tenenbaum [14]). The function $\Phi$ was first studied by Buchstab [3] and later by de Bruijn [1]. For the estimate on $\Phi$ below see also Theorem III.6.3 in Tenenbaum [14].

THEOREM 1.1. Uniformly for $x \geq z \geq 2$, we have

$$
\Psi(x, z)=x \varrho(v)+O\left(\frac{x \log z}{\log ^{2} x}\right) .
$$

TheOREM 1.2. Uniformly for $x \geq y \geq 2$, we have

$$
\Phi(x, y)=\frac{x \omega(u)-y}{\log y}+O\left(\frac{x}{\log ^{2} y}\right) .
$$

Theorems 1.1 and 1.2 allow us to derive an asymptotic formula for $\Gamma(x, y, z)$. This formula involves a function $\eta(u, v)$, defined for $0<u \leq v$ by

$$
\eta(u, v):=\varrho(v)+\int_{0}^{u} \varrho(t v / u) \omega(u-t) d t \quad(0<u \leq v) .
$$

For $0<u \leq 1$, the integral vanishes and we have $\eta(u, v)=\varrho(v)$. We extend the definition of $\eta(u, v)$ to all real values of $u$ and $v$ by setting $\eta(0, v):=\varrho(v)$, $\eta(u, v):=0$ for $u<0$ or $v<0$ and finally $\eta(u, v)=1$ for $0 \leq v<u$. Note that $\eta(u, v)$ is continuous for $0<u<v$ since $\varrho(v)$ is continuous for $v>0$ and the integrand is uniformly bounded. The function $\eta(u, v)$ is also continuous at $0<u=v$ due to the well known convolution identity (see [14], p. 420)

$$
1=\varrho(u)+\int_{0}^{u} \varrho(t) \omega(u-t) d t .
$$

Also, $\eta(u, v)>0$ for $0 \leq u \leq v$.

A function closely related to $\Gamma(x, y, z)$ is the function $\theta(x, y, z)$, which denotes the number of positive integers not exceeding $x$ all of whose prime 
divisors are in the interval $(z, y]$. The latter function has been studied by Friedlander [6] and, more recently, by Saias [10-12]. Like $\Gamma(x, y, z), \theta(x, y, z)$ is also a generalization of the functions $\Psi$ and $\Phi$.

The function

$$
\sigma(u, v):=\frac{u}{v} \varrho^{\prime}(u)+\int_{0}^{\infty} \varrho\left(u-\frac{u}{v} t\right) d \omega(t) \quad(v \geq u>0, u \neq 1)
$$

arises in the study of $\theta(x, y, z)$. For results on $\sigma(u, v)$ see Friedlander [6] and Saias [10].

In this paper we establish an asymptotic estimate for $\Gamma(x, y, z)$ by expressing this function in terms of the functions $\Psi$ and $\Phi$ and estimating the latter functions by means of Theorems 1.1 and 1.2. In a sequel to this paper (see [15]), we will use complex integration together with the saddle-point method to sharpen many of the results obtained here.

2. Some observations. Let $P=\prod_{z<p \leq y} p$. The Möbius inversion formula allows us to write the characteristic function of the set of integers $n$ which are free of prime divisors from the interval $(z, y]$ as

$$
\sum_{\substack{d|P \\ d| n}} \mu(d)
$$

Summing over $n \leq x$, we obtain

$$
\begin{aligned}
\Gamma(x, y, z) & =\sum_{d \mid P} \mu(d)\left[\frac{x}{d}\right]=\sum_{d \mid P} \mu(d) \frac{x}{d}-\sum_{d \mid P} \mu(d)\left\{\frac{x}{d}\right\} \\
& =x \prod_{z<p \leq y}\left(1-\frac{1}{p}\right)-\sum_{d \mid P} \mu(d)\left\{\frac{x}{d}\right\} .
\end{aligned}
$$

Let us first consider the trivial estimate

$$
\left|\sum_{d \mid P} \mu(d)\left\{\frac{x}{d}\right\}\right| \leq \sum_{d \mid P} 1 \leq 2^{y-z} .
$$

If $y-z \leq \log x$ we have $2^{y-z} \leq x^{\log 2}<x^{3 / 4}$. The main term in (3) is $\gg x / \log x$, by Mertens' formula. This gives

$$
\Gamma(x, y, z) \sim x \prod_{z<p \leq y}\left(1-\frac{1}{p}\right)
$$

for $y-z \leq \log x$.

With $u=\log x / \log y$, the fundamental lemma of the combinatorial sieve (see [14], Theorem I.4.3) implies that, uniformly for $x \geq y \geq z \geq 3 / 2$, 


$$
\Gamma(x, y, z)=x \prod_{z<p \leq y}\left(1-\frac{1}{p}\right)\left\{1+O\left(u^{-u / 2}\right)\right\}+O(\theta(x, y, z)) .
$$

Since $\theta(x, y, z) \leq \Psi(x, y) \ll x e^{-u / 2}$ (see Theorem III.5.1 of [14]), it follows from (5) that (4) holds in the domain $1 \leq z \leq y \leq \exp \left\{c \log x / \log _{2} x\right\}$, for some suitable constant $c$.

It is easy to see, however, that (4) does not hold uniformly for $1 \leq z \leq$ $y \leq \sqrt{x}$. To this end choose $z=1$ and $y=\sqrt{x}$. Then the prime number theorem gives

$$
\Gamma(x, y, z) \sim \frac{x}{\log x},
$$

but Mertens' formula shows that

$$
x \prod_{1<p \leq \sqrt{x}}\left(1-\frac{1}{p}\right) \sim \frac{2 x e^{-\gamma}}{\log x},
$$

where $\gamma$ denotes Euler's constant.

3. An asymptotic formula for $\Gamma(x, y, z)$. In this section we will derive the following result for $\Gamma(x, y, z)$.

TheOREM 3.1. Uniformly for $x \geq y \geq z \geq 3 / 2$, we have

$$
\Gamma(x, y, z)=x \eta(u, v)+O\left(\frac{x}{\log y}\right) .
$$

Theorem 3.1 and the lower bound on $\eta(u, v)$ in Lemma 3.4 (see below) will allow us to deduce the following asymptotic result.

Theorem 3.2. Uniformly for $y \geq z \geq 3 / 2$, we have

$$
\Gamma(x, y, z)= \begin{cases}x \eta(u, v)\left\{1+O\left(\frac{1}{\log z}\right)\right\} & \text { for } x \geq y z, \\ x \eta(u, v)\left\{1+O\left(\frac{1}{\log (x / y)+\varrho(v) \log x}\right)\right\} & \text { for } y \leq x<y z .\end{cases}
$$

The proof of Theorem 3.1 is based on the following identity for $\Gamma(x, y, z)$, which allows us to derive the result from the known estimates for $\Psi(x, z)$ and $\Phi(x, y)$ given in Theorems 1.1 and 1.2.

Lemma 3.3. For $x \geq y \geq z \geq 1$ we have

$$
\Gamma(x, y, z)=\Psi(x, z)-\Psi(x / y, z)+\sum_{\substack{n \leq x / y \\ P^{+}(n) \leq z}} \Phi(x / n, y) .
$$

Proof. If the integer $m \geq 1$ is counted in $\Gamma(x, y, z)$, we can write $m=n d$ with $P^{+}(n) \leq z$ and $P^{-}(d)>y$. For each $n \leq x / y$ there are $\Phi(x / n, y)$ 
possible choices for $d$. If $x / y<n \leq x$ then $d=1$ is the only choice and the contribution from these $n$ is $\Psi(x, z)-\Psi(x / y, z)$.

We are now ready to prove Theorem 3.1.

Proof of Theorem 3.1. The result being trivial for bounded $y$, we assume that $y>y_{0}$ for a sufficiently large constant $y_{0}$. If $x \leq y z$, it follows from Lemma 3.3 and Theorems 1.1 and 1.2 that

$$
\begin{aligned}
\Gamma(x, y, z)= & \Psi(x, z)-\Psi(x / y, z)+\sum_{n \leq x / y} \Phi(x / n, y) \\
= & x \varrho(v)+O\left(\frac{x}{\log y}\right) \\
& +\sum_{n \leq x / y}\left\{\frac{\frac{x}{n} \omega\left(\frac{\log (x / n)}{\log y}\right)-y}{\log y}+O\left(\frac{x / n}{\log ^{2} y}\right)\right\} .
\end{aligned}
$$

Hence

$$
\Gamma(x, y, z)=x \varrho(v)+\sum_{n \leq x / y} \frac{x \omega\left(\frac{\log (x / n)}{\log y}\right)}{n \log y}+O\left(\frac{x}{\log y}\right),
$$

for $x \leq y z$. Now $\omega(\log (x / t) / \log y) / t$ is a monotonic function in $t$, since

$$
\left(\frac{\omega\left(\frac{\log (x / t)}{\log y}\right)}{t}\right)^{\prime}=\frac{1}{t^{2}}\left(-\omega^{\prime}\left(\frac{\log (x / t)}{\log y}\right)(\log y)^{-1}-\omega\left(\frac{\log (x / t)}{\log y}\right)\right)<0
$$

for $y>y_{0}$. Thus, we can write

$$
\sum_{n \leq x / y} \frac{x \omega\left(\frac{\log (x / n)}{\log y}\right)}{n \log y}=\int_{1}^{x / y} \frac{x \omega\left(\frac{\log (x / t)}{\log y}\right)}{t \log y} d t+O\left(\frac{x}{\log y}\right) .
$$

For $0 \leq v \leq 1$ we have $\varrho(v)=1$. Thus (7) and (8) imply, for $x \leq y z$,

$$
\begin{aligned}
\Gamma(x, y, z) & =x \varrho(v)+\int_{1}^{x / y} \frac{x \omega\left(\frac{\log (x / t)}{\log y}\right)}{t \log y} \varrho\left(\frac{\log t}{\log z}\right) d t+O\left(\frac{x}{\log y}\right) \\
& =x \varrho(v)+x \int_{0}^{u-1} \omega(u-t) \varrho(t v / u) d t+O\left(\frac{x}{\log y}\right) \\
& =x \eta(u, v)+O\left(\frac{x}{\log y}\right) .
\end{aligned}
$$

In the remainder of the proof we establish the theorem for $x>y z$. From Lemma 3.3 and Theorem 1.1 we have

$$
\Gamma(x, y, z)=x \varrho(v)+\sum_{\substack{n \leq x / y \\ P^{+}(n) \leq z}} \Phi(x / n, y)+O\left(\frac{x}{\log y}\right) .
$$


Theorem 1.2 implies

$$
\begin{aligned}
\sum_{\substack{n \leq x / y \\
P^{+}(n) \leq z}} \Phi(x / n, y) & =\sum_{\substack{n \leq x / y \\
P^{+}(n) \leq z}}\left\{\frac{\frac{x}{n} \omega\left(\frac{\log (x / n)}{\log y}\right)-y}{\log y}+O\left(\frac{x / n}{\log ^{2} y}\right)\right\} \\
& =\int_{1}^{x / y} \frac{x \omega\left(\frac{\log (x / t)}{\log y}\right)}{t \log y} d \Psi(t, z)+O\left(\frac{x}{\log y}\right) .
\end{aligned}
$$

By Theorem 1.1, we have

$$
\begin{aligned}
\int_{1}^{x / y} \frac{x \omega\left(\frac{\log (x / t)}{\log y}\right)}{t \log y} d \Psi(t, z) & \\
= & \int_{1}^{z} \frac{x \omega\left(\frac{\log (x / t)}{\log y}\right)}{t \log y} d[t]+\int_{z}^{x / y} \frac{x \omega\left(\frac{\log (x / t)}{\log y}\right)}{t \log y} d\left\{t \varrho\left(\frac{\log t}{\log z}\right)+E(t)\right\} \\
= & \int_{1}^{z} \frac{x \omega\left(\frac{\log (x / t)}{\log y}\right)}{t \log y} \varrho\left(\frac{\log t}{\log z}\right) d(t-\{t\})+\int_{z}^{x / y} \frac{x \omega\left(\frac{\log (x / t)}{\log y}\right)}{t \log y} \varrho\left(\frac{\log t}{\log z}\right) d t \\
& +\int_{z}^{x / y} \frac{x \omega\left(\frac{\log (x / t)}{\log y}\right)}{t \log y} \cdot \frac{\varrho^{\prime}\left(\frac{\log t}{\log z}\right)}{\log z} d t+\int_{z}^{x / y} \frac{x \omega\left(\frac{\log (x / t)}{\log y}\right)}{t \log y} d E(t)
\end{aligned}
$$

where $E(t)=O\left((t \log z) / \log ^{2} t\right)$.

Use integration by parts on the first and last integral, noting that $\omega$ and $\omega^{\prime}$ are uniformly bounded, and observe that

$$
\int_{1}^{x / y} \frac{x \omega\left(\frac{\log (x / t)}{\log y}\right)}{t \log y} \varrho\left(\frac{\log t}{\log z}\right) d t=x \int_{0}^{u-1} \omega(u-t) \varrho(t v / u) d t=x(\eta(u, v)-\varrho(v)) .
$$

We obtain

$$
\begin{aligned}
& \int_{1}^{x / y} \frac{x \omega\left(\frac{\log (x / t)}{\log y}\right)}{t \log y} d \Psi(t, z) \\
& =x \eta(u, v)-x \varrho(v)+O\left(\frac{x}{\log y}\right)+O\left(\frac{x}{\log y} \int_{z}^{x / y} \frac{\varrho^{\prime}\left(\frac{\log t}{\log z}\right)}{t \log z} d t\right) \\
& +O\left(\frac{x}{\log y} \int_{z}^{x / y} \frac{t \log z}{\log ^{2} t} d\left\{\frac{\omega\left(\frac{\log (x / t)}{\log y}\right)}{t}\right\}\right)
\end{aligned}
$$




$$
\begin{aligned}
= & x \eta(u, v)-x \varrho(v)+O\left(\frac{x}{\log y}\right)+O\left(\left.\frac{x}{\log y} \varrho\left(\frac{\log t}{\log z}\right)\right|_{z} ^{x / y}\right) \\
& +O\left(\frac{x}{\log y} \int_{z}^{x / y} \frac{t \log z}{\log ^{2} t} \cdot \frac{1}{t^{2}} d t\right) .
\end{aligned}
$$

Thus,

$$
\int_{1}^{x / y} \frac{x \omega\left(\frac{\log (x / t)}{\log y}\right)}{t \log y} d \Psi(t, z)=x \eta(u, v)-x \varrho(v)+O\left(\frac{x}{\log y}\right) .
$$

So (10) and (11) show that for $x>y z$ we have

$$
\sum_{\substack{n \leq x / y \\ P^{+}(n) \leq z}} \Phi(x / n, y)=x \eta(u, v)-x \varrho(v)+O\left(\frac{x}{\log y}\right) .
$$

Together with (9) this gives

$$
\Gamma(x, y, z)=x \eta(u, v)+O\left(\frac{x}{\log y}\right)
$$

for $x>y z$, and hence for all $x \geq y \geq z \geq 3 / 2$. This completes the proof of Theorem 3.1.

LEMMA 3.4. If $u \leq v$, then

$$
\begin{array}{ll}
\eta(u, v) \leq e^{\gamma} \frac{u}{v} & \text { for } u \geq 1, \\
\eta(u, v) \geq \frac{u}{2 v} & \text { for } u \geq \frac{v}{v-1} .
\end{array}
$$

Proof. (i) Since $\omega(u) \leq 1$ we have, for $u \geq 1$,

$$
\begin{aligned}
\eta(u, v) & =\varrho(v)+\int_{0}^{u-1} \varrho(t v / u) \omega(u-t) d t \leq \varrho(v)+\int_{0}^{u-1} \varrho(t v / u) d t \\
& =\varrho(v)+\frac{u}{v} \int_{0}^{v(1-1 / u)} \varrho(t) d t \leq \varrho(v)+\frac{u}{v} e^{\gamma}-\frac{u}{v} \int_{v(1-1 / u)}^{\infty} \varrho(t) d t \\
& \leq \varrho(v)+\frac{u}{v} e^{\gamma}-u \frac{1}{v} \int_{v-1}^{v} \varrho(t) d t=\frac{u}{v} e^{\gamma}+\varrho(v)-u \varrho(v) \leq \frac{u}{v} e^{\gamma} .
\end{aligned}
$$

(ii) Since $\omega(u) \geq 1 / 2$ for $u \geq 1$, we have, for $u \geq v /(v-1)$,

$$
\eta(u, v) \geq \int_{0}^{u-1} \varrho(t v / u) \omega(u-t) d t \geq \frac{u}{2 v} \int_{0}^{v-v / u} \varrho(t) d t \geq \frac{u}{2 v} \int_{0}^{1} \varrho(t) d t=\frac{u}{2 v}
$$


Proof of Theorem 3.2. If $y \leq x<y z$, then $1 \leq u<v /(v-1)$ and

$$
\begin{aligned}
\eta(u, v) & =\varrho(v)+\int_{0}^{u} \varrho(t v / u) \omega(u-t) d t=\varrho(v)+\int_{0}^{u-1} \frac{1}{u-t} d t \\
& =\varrho(v)+\log u>\varrho(v)+(u-1) / 2,
\end{aligned}
$$

which gives

$$
\eta(u, v) \log y>(\log y) \varrho(v)+\frac{\log (x / y)}{2}>\frac{1}{2}((\log x) \varrho(v)+\log (x / y)) .
$$

If $x \geq y z$ then $u \geq v /(v-1)$ and Lemma 3.4 shows that

$$
\eta(u, v) \log y \geq(\log y) u /(2 v)=(\log z) / 2 .
$$

Theorem 3.1 yields

$$
\Gamma(x, y, z)=x \eta(u, v)\left\{1+O\left(\frac{1}{\eta(u, v) \log y}\right)\right\},
$$

which concludes the proof of Theorem 3.2.

4. The difference-differential equations for $\eta(u, v)$. Like $\Psi(x, z)$ and $\Phi(x, y), \Gamma(x, y, z)$ also satisfies functional equations.

Proposition 4.1. (i) Let $z \leq y_{1} \leq y_{2} \leq x$. Then

$$
\Gamma\left(x, y_{1}, z\right)-\Gamma\left(x, y_{2}, z\right)=\sum_{y_{1}<p \leq y_{2}} \Gamma(x / p, p-1, z) .
$$

(ii) Let $z_{1} \leq z_{2}<y<x$. Then

$$
\Gamma\left(x, y, z_{2}\right)-\Gamma\left(x, y, z_{1}\right)=\sum_{z_{1}<p \leq z_{2}} \Gamma(x / p, y, p) .
$$

Proof. Part (i) follows from grouping the integers contributing to the left hand side according to their smallest prime divisor in $\left[y_{1}, x\right]$. Part (ii) follows from grouping the integers contributing to the left hand side according to their largest prime divisor in $\left[2, z_{2}\right]$.

The smooth versions of the functional equations in Proposition 4.1 are the following difference-differential equations.

Proposition 4.2. For $1<u<v$ the partial derivatives $\partial \eta / \partial u$ and $\partial \eta / \partial v$ are continuous and satisfy

$$
u \frac{\partial}{\partial u} \eta(u, v)=\eta\left(u-1, v\left(1-u^{-1}\right)\right)
$$

and

$$
v \frac{\partial}{\partial v} \eta(u, v)=-\eta\left(u\left(1-v^{-1}\right), v-1\right) .
$$


Proof. From the definition of $\eta(u, v)$ in (1) we have

$$
\eta(u, v)=\varrho(v)+\int_{0}^{1} \varrho(v(1-t)) u \omega(u t) d t .
$$

Hence

$$
\begin{aligned}
\frac{\partial}{\partial u} \eta(u, v) & =\frac{1}{u} \int_{0}^{1} \varrho(v(1-t)) d(u t \omega(u t)) \\
& =\frac{1}{u} \varrho\left(v\left(1-u^{-1}\right)\right)+\int_{1 / u}^{1} \varrho(v(1-t)) \omega(u t-1) d t \\
& =\frac{1}{u} \varrho\left(v\left(1-u^{-1}\right)\right)+\frac{1}{u} \int_{0}^{u-1} \varrho(t v / u) \omega(u-1-t) d t \\
& =\frac{1}{u} \eta\left(u-1, v\left(1-u^{-1}\right)\right),
\end{aligned}
$$

which proves (12). To show (13) we write

$$
\begin{aligned}
v \frac{\partial}{\partial v} \eta(u, v) & =v \varrho^{\prime}(v)+v \int_{0}^{u} \varrho^{\prime}(t v / u) t u^{-1} \omega(u-t) d t \\
& =-\varrho(v-1)-\int_{0}^{u} \varrho(t v / u-1) \omega(u-t) d t \\
& =-\varrho(v-1)-\int_{0}^{u\left(1-v^{-1}\right)} \varrho(t v / u) \omega\left(u\left(1-v^{-1}\right)-t\right) d t \\
& =-\eta\left(u\left(1-v^{-1}\right), v-1\right) .
\end{aligned}
$$

REMARK 4.3. Note that we could have defined $\eta(u, v)$ for $0<u<v$ as the unique continuous solution of the difference-differential equation (12) for $u>1$ together with the initial condition $\eta(u, v)=\varrho(v)$ for $0<u \leq 1$. The natural way to obtain an asymptotic formula for $\Gamma(x, y, z)$ would then be to employ the functional equation in Proposition 4.1 and then induct on the variable $u$. However, the explicit definition in (1) together with Lemma 3.3 allows us to make direct use of well known results on $\Psi(x, z)$ and $\Phi(x, y)$.

REMARK 4.4. With the definition of $\eta(u, v)$ extended to the whole $(u, v)$ plane, one easily verifies that the difference-differential equations (12) and (13) hold for all $u, v \in \mathbb{R}$ except for $u \in\{0,1\}, v \in\{0,1\}$ or $u=v$.

In the following we will use the notation $\eta_{r}(u)=\eta(u, u / r)$ for $0<r \leq 1$. For $r=0$ we define $\eta_{0}(u)=0$. 
Lemma 4.5. Let $\eta_{r}^{\prime}(u)=\frac{\partial}{\partial u} \eta_{r}(u)$. For $0 \leq r \leq 1$ and $u>1$ we have

$$
u \eta_{r}^{\prime}(u)=\eta_{r}(u-1)-\eta_{r}(u-r) .
$$

Proof. The result is trivial when $r=0,1$ since both sides vanish. By the previous results $\partial \eta / \partial u$ and $\partial \eta / \partial v$ exist and are continuous for $v>u>1$. The result follows from the chain rule.

By differentiating (14) $n-1$ times we obtain

Corollary 4.6. Let $0 \leq r \leq 1$ and let

$$
D_{n, r}=\{i+j r: i, j \geq 0, i+j \leq n\} .
$$

Then $\eta_{r}(u)$ is $n$ times continuously differentiable on $\mathbb{R} \backslash D_{n, r}$. Furthermore

$$
\begin{array}{r}
u \eta_{r}^{(n)}(u)=\eta_{r}^{(n-1)}(u-1)-\eta_{r}^{(n-1)}(u-r)-(n-1) \eta_{r}^{(n-1)}(u) \\
\text { for } n \geq 1,0 \leq r \leq 1, u \in \mathbb{R} \backslash D_{n, r} .
\end{array}
$$

We define $\eta_{r}^{(n)}(u)$ on $D_{n, r}$ by right continuity. Then (15) is valid for all $u \in \mathbb{R}$.

\section{Results on $\eta(u, v)$ derived from the difference-differential equations}

Lemma 5.1. We have, for $0 \leq r \leq 1$,

$$
\begin{aligned}
& \lim _{u \rightarrow \infty} \eta_{r}(u)=r, \\
& \lim _{u \rightarrow \infty} \eta_{r}^{(n)}(u)=0 \quad \text { for } n \geq 1 .
\end{aligned}
$$

Proof. If $r=0$, then (i) is trivial. Let $0<r \leq 1$. Now $\omega(u) \rightarrow e^{-\gamma}$ and $\varrho(u) \rightarrow 0$ as $u \rightarrow \infty$. Thus

$$
\begin{aligned}
\eta_{r}(u) & =\varrho(u / r)+\int_{0}^{u} \varrho(t / r) \omega(u-t) d t \\
& =e^{-\gamma} \int_{0}^{u} \varrho(t / r) d t+o(1)=r e^{-\gamma} \int_{0}^{u / r} \varrho(t) d t+o(1)
\end{aligned}
$$

as $u$ tends to infinity. Part (i) follows, since $\int_{0}^{\infty} \varrho(t) d t=e^{\gamma}$ by Theorem III.5.7 in [14]. Part (ii) follows from (i) and Corollary 4.6 by induction on $n$.

With $\sigma_{r}(u):=\sigma(u, u / r), 0<r \leq 1$ it follows from the definition (2) of $\sigma(u, v)$ that, for $0 \leq r \leq 1, u>1, u \neq 2 r, 1+r, 2$,

$$
\left(u \sigma_{r}(u)\right)^{\prime}=\sigma_{r}(u-r)-\sigma_{r}(u-1) .
$$

This allows us to bound $\eta_{r}^{\prime}(u)$ in terms of $\sigma_{r}(u)$. The behaviour of $\sigma_{r}(u)$ is well understood due to the work of Friedlander [6] and Saias [10]. 
LEMma 5.2. For $1 \leq u \leq 3$ and $r \leq 1 / 3$ we have $\sigma_{r}(u) \gg 1$.

Proof. This follows directly from Theorem 2 and Theorem 5 in [6].

Proposition 5.3. Let $\eta_{r}^{\prime}=\partial \eta_{r} / \partial u$. Then, for $0 \leq r \leq 1$,

$$
\begin{array}{ll}
\eta_{r}^{\prime}(u) \ll r \sigma_{r}(u) & \text { for } u \geq 2, \\
\eta_{r}^{\prime}(u) \ll \sigma_{r}(u) & \text { for } u>1 .
\end{array}
$$

Proof. (i) We first consider the case $r \leq 1 / 3$. For $2 \leq u \leq 3$ and $r \leq 1 / 3$ we have $\sigma_{r}(u) \gg 1$, by Lemma 5.2. Lemmas 4.5 and 3.4 imply $\eta_{r}^{\prime}(u)=$ $\left(\eta_{r}(u-1)-\eta_{r}(u-r)\right) / u \ll r$ for $2 \leq u \leq 3$. Thus we have $\eta_{r}^{\prime}(u) \ll r \sigma_{r}(u)$ for $2 \leq u \leq 3$ and $r \leq 1 / 3$. Now assume that $\eta_{r}^{\prime}(u) \leq \operatorname{cr} \sigma_{r}(u)$ for some positive constant $c$ and $u \leq N$, where $N \geq 3$. For $N<u \leq N+r$ we have

$$
\left|\eta_{r}^{\prime}(u)\right|=\left|\frac{1}{u} \int_{u-1}^{u-r} \eta_{r}^{\prime}(t) d t\right| \leq \frac{c r}{u}\left|\int_{u-1}^{u-r} \sigma_{r}(t) d t\right|=\operatorname{cr} \sigma_{r}(u),
$$

which proves (i) in the case $r \leq 1 / 3$.

Assume $r>1 / 3$. For $0<u \leq 1$ we have $\sigma_{r}(u)=\sigma(u, v)=\omega(v)$ from the definition of $\sigma(u, v)$ in (2). Also, $\varrho^{\prime}(v) \ll \omega(v)$. Indeed, $\varrho^{\prime}(v)=0=\omega(v)$ for $0 \leq v<1$, and $\left|\varrho^{\prime}(v)\right|=\varrho(v-1) / v \leq 1 / v$ and $1 / 2 \leq \omega(v)$ for $1 \leq v$. Thus

$$
\eta_{r}^{\prime}(u)=\frac{1}{r} \varrho^{\prime}\left(\frac{u}{r}\right) \leq 3 \varrho^{\prime}(v) \ll \omega(v)=\sigma_{r}(u) \leq r \sigma_{r}(u) .
$$

Now assume that $\eta_{r}^{\prime}(u) \leq \operatorname{cr} \sigma_{r}(u)$ for some positive constant $c$ and $u \leq N$, where $N \geq 1$. For $N<u \leq N+r$ we have

$$
\left|\eta_{r}^{\prime}(u)\right|=\left|\frac{1}{u} \int_{u-1}^{u-r} \eta_{r}^{\prime}(t) d t\right| \leq \frac{c r}{u}\left|\int_{u-1}^{u-r} \sigma_{r}(t) d t\right|=\operatorname{cr} \sigma_{r}(u) .
$$

This shows that, in the case $r>1 / 3$, we have $\eta_{r}^{\prime}(u) \ll r \sigma_{r}(u)$ for $u>0$, which completes the proof of part (i).

(ii) In view of (i), it suffices to consider $1<u<2$ and $r \leq 1 / 3$. We have $\sigma_{r}(u) \gg 1$, by Lemma 5.2. On the other hand we clearly have $\eta_{r}^{\prime}(u) \ll 1$ for $u>1$. This concludes the proof of Proposition 5.3.

Corollary 5.4. For $u \geq 1,0 \leq r \leq 1$, we have

$$
\begin{array}{ll}
\eta_{r}(u)=r\left(1+O\left(\int_{u}^{\infty} \sigma_{r}(t) d t\right)\right) & \text { for } u \geq 2, \\
\eta_{r}(u)=r(1+O(\varrho(u))) & \text { for } u \geq 1 .
\end{array}
$$


Proof. (i) follows from Proposition 5.3 and Lemma 5.1.

(ii) For $u \geq 2$ we have

$$
\int_{u}^{\infty} \sigma_{r}(t) d t \ll \int_{u}^{\infty} \varrho(t) d t \ll \varrho(u) .
$$

If $1 \leq u<2$, (ii) follows from Lemma 3.4.

Theorem 5.5. We have, for $0<u \leq v$,

$$
\eta(u, v)=e^{\gamma} \omega(u) \frac{u}{v}+O\left(\frac{\log v}{v^{2}} \max \left(1, \frac{1}{u-1}, u-1\right)\right) .
$$

Proof. For $0<u \leq 1$ the left hand side is equal to $\varrho(v)<1 / \Gamma(v+1)=$ $O\left(1 / v^{2}\right)$. On the right side we have $\omega(u)=0$ in this case. For $1<u \leq 2$ we have

$$
\begin{aligned}
\eta(u, v) & =\varrho(v)+\int_{0}^{u-1} \varrho(t v / u) \frac{d t}{u-t}=\varrho(v)+\int_{0}^{v(1-1 / u)} \varrho(s) \frac{d s}{v-s} \\
& =\varrho(v)+\int_{0}^{\log v} \varrho(s) \frac{d s}{v-s}+\int_{\log v}^{v(1-1 / u)} \varrho(s) \frac{d s}{v-s} \\
& \leq \varrho(v)+\frac{1}{v-\log v} e^{\gamma}+\frac{1}{v / 2} \int_{\log v}^{\infty} \varrho(s) d s \\
& \leq \varrho(v)+\frac{e^{\gamma}}{v}+e^{\gamma} \frac{\log v}{v(v-\log v)}+O\left(\frac{1}{v^{2}}\right) \leq \frac{e^{\gamma}}{v}+O\left(\frac{\log v}{v^{2}}\right) .
\end{aligned}
$$

On the other hand, we have

$$
\begin{aligned}
\eta(u, v) & =\varrho(v)+\int_{0}^{v(1-1 / u)} \varrho(s) \frac{d s}{v-s} \geq \varrho(v)+\frac{1}{v} \int_{0}^{v(1-1 / u)} \varrho(s) d s \\
& =\varrho(v)+\frac{e^{\gamma}}{v}-\frac{1}{v} \int_{v(1-1 / u)}^{\infty} \varrho(s) d s \geq \varrho(v)+\frac{e^{\gamma}}{v}-\frac{1}{v^{2}(1-1 / u)} \\
& \geq \varrho(v)+\frac{e^{\gamma}}{v}-\frac{1}{v^{2}(u-1)} .
\end{aligned}
$$

This proves the result for $1<u \leq 2$.

For $2<u \leq 3$ we use (12) to write

$$
\eta(u, v)=\eta(2, v)+\int_{2}^{u} \eta\left(t-1, v\left(1-t^{-1}\right)\right) \frac{d t}{t}
$$




$$
\begin{aligned}
& =e^{\gamma} \omega(2) \frac{2}{v}+O\left(\frac{\log v}{v^{2}}\right)+\int_{2}^{2+2(\log v) / v} \eta\left(t-1, v\left(1-t^{-1}\right)\right) \frac{d t}{t} \\
& +\int_{2+2(\log v) / v}^{u} \eta\left(t-1, v\left(1-t^{-1}\right)\right) \frac{d t}{t} .
\end{aligned}
$$

From Lemma 3.4 we know that $\eta(u, v)=O(u / v)$. Thus,

$$
\int_{2}^{2+2(\log v) / v} \eta\left(t-1, v\left(1-t^{-1}\right)\right) \frac{d t}{t}=O\left(\frac{\log v}{v^{2}}\right) .
$$

Next, note that $t \geq 2+2(\log v) / v$ implies

$$
v\left(1-\frac{1}{t}\right)\left(1-\frac{1}{t-1}\right) \geq \log \left(v\left(1-\frac{1}{t}\right)\right) .
$$

Thus, for $t \geq 2+2(\log v) / v$,

$$
\begin{aligned}
\eta\left(t-1, v\left(1-t^{-1}\right)\right) \geq & \frac{e^{\gamma}}{v\left(1-t^{-1}\right)}+\varrho\left(v\left(1-t^{-1}\right)\right) \\
& -\frac{1}{v\left(1-t^{-1}\right)} \int_{\log \left(v\left(1-t^{-1}\right)\right)}^{\infty} \varrho(s) d s \\
\geq & \frac{e^{\gamma}}{v\left(1-t^{-1}\right)}+O\left(1 / v^{2}\right) .
\end{aligned}
$$

Together with the upper bound for $\eta(u, v)$ in the region $1<u \leq 2$ this implies, for $t \geq 2+2(\log v) / v$,

$$
\eta\left(t-1, v\left(1-t^{-1}\right)\right)=\frac{e^{\gamma}}{v\left(1-t^{-1}\right)}+O\left(\frac{\log \left(v\left(1-t^{-1}\right)\right)}{v^{2}\left(1-t^{-1}\right)^{2}}\right) .
$$

Hence,

$$
\begin{aligned}
\int_{2+2(\log v) / v}^{u} \eta(t- & \left.1, v\left(1-t^{-1}\right)\right) \frac{d t}{t} \\
& =\int_{2+2(\log v) / v}^{u}\left(\frac{e^{\gamma}}{v\left(1-t^{-1}\right)}+O\left(\frac{\log v}{v^{2}}\right)\right) \frac{d t}{t} \\
& =\frac{e^{\gamma}}{v}\left(\log (u-1)-\log \left(1+\frac{2 \log v}{v}\right)\right)+O\left(\frac{\log v}{v^{2}}\right) \\
& =\frac{e^{\gamma}}{v} \log (u-1)+O\left(\frac{\log v}{v^{2}}\right) .
\end{aligned}
$$


Therefore,

$$
\eta(u, v)=\frac{e^{\gamma}}{v}(1+\log (u-1))+O\left(\frac{\log v}{v^{2}}\right)=e^{\gamma} \frac{u}{v} \omega(u)+O\left(\frac{\log v}{v^{2}}\right)
$$

in the region $2<u \leq 3$.

For $u>3$ we proceed by induction. Let $N \geq 3$ and assume that, for $N-1<u \leq N$, there exists a constant $c$ such that

$$
\left|\eta(u, v)-e^{\gamma} \omega(u) \frac{u}{v}\right| \leq c \frac{(N-2) \log v}{v^{2}} .
$$

Then, for $N<u \leq N+1$,

$$
\begin{aligned}
&\left|\eta(u, v)-e^{\gamma} \omega(u) \frac{u}{v}\right| \\
&=\left|\eta(N, v)+\int_{N}^{u} \eta\left(t-1, v\left(1-t^{-1}\right)\right) \frac{d t}{t}-e^{\gamma} \omega(u) \frac{u}{v}\right| \\
&=\mid \eta(N, v)-e^{\gamma} \omega(N) \frac{N}{v} \\
&+\int_{N}^{u}\left(\eta\left(t-1, v\left(1-t^{-1}\right)\right)-e^{\gamma} \omega(t-1) \frac{t-1}{v\left(1-t^{-1}\right)}\right) \frac{d t}{t} \\
&+\int_{N}^{u} \frac{e^{\gamma}}{v} \omega(t-1) d t+e^{\gamma} \omega(N) \frac{N}{v}-e^{\gamma} \omega(u) \frac{u}{v} \mid .
\end{aligned}
$$

Since $\omega(u)$ satisfies $(u \omega(u))^{\prime}=\omega(u-1)$, the last three terms in the previous expression combine to zero and we complete the proof of Theorem 5.5 as follows:

$$
\begin{aligned}
\left|\eta(u, v)-e^{\gamma} \omega(u) \frac{u}{v}\right| \leq & \left|\eta(N, v)-e^{\gamma} \omega(N) \frac{N}{v}\right| \\
& +\int_{N}^{u}\left|\eta\left(t-1, v\left(1-t^{-1}\right)\right)-e^{\gamma} \omega(t-1) \frac{t-1}{v\left(1-t^{-1}\right)}\right| \frac{d t}{t} \\
\leq & c \frac{(N-2) \log v}{v^{2}}+c \int_{N}^{u} \frac{(N-2) \log \left(v\left(1-t^{-1}\right)\right)}{v^{2}\left(1-t^{-1}\right) t} d t \\
\leq & c \frac{(N-2) \log v}{v^{2}}\left(1+\int_{N}^{u} \frac{d t}{t-2+t^{-1}}\right) \\
\leq & c \frac{(N-2) \log v}{v^{2}}\left(1+\frac{1}{N-2}\right)=c \frac{(N-1) \log v}{v^{2}} .
\end{aligned}
$$




\section{References}

[1] N. G. de Bruijn, On the number of uncancelled elements in the sieve of Eratosthenes, Proc. Konink. Nederl. Akad. Wetensch. 53 (1950), 803-812.

[2] - On the number of positive integers $\leq x$ and free of prime factors $>y$, ibid. 54 (1951), 50-60.

[3] A. A. Buchstab, An asymptotic estimation of a general number-theoretic function, Mat. Sb. (2) 44 (1937), 1239-1246.

[4] H. Daboussi, On a convolution method, in: E. Aparicio, C. Calderón and J. C. Peral (eds.), Congreso de Teoria de los Números (Universitad del País Vasco), 1989, 110-137.

[5] H. Davenport and P. Erdös, The distribution of quadratic and higher residues, Publ. Math. Debrecen 2 (1952), 252-265.

[6] J. B. Friedlander, Integers free from small and large primes, Proc. London Math. Soc. (3) 33 (1976), 565-576.

[7] W. B. Jurkat and H. E. Richert, An improvement of Selberg's sieve method I, Acta Arith. 11 (1965), 217-240.

[8] R. A. Rankin, The difference between consecutive prime numbers, J. London Math. Soc. 13 (1938), 242-247.

[9] -, The difference between consecutive prime numbers V, Proc. Edinburgh Math. Soc. (2) 13 (1963), 331-332.

[10] E. Saias, Entiers sans grand ni petit facteur premier I, Acta Arith. 61 (1992), $347-374$.

[11] —, Entiers sans grand ni petit facteur premier II, ibid. 63 (1993), 287-312.

[12] —, Entiers sans grand ni petit facteur premier III, ibid. 71 (1995), 351-379.

[13] A. Schönhage, Eine Bemerkung zur Konstruktion großer Primzahllücken, Arch. Math. (Basel) 14 (1963), 29-30.

[14] G. Tenenbaum, Introduction to Analytic and Probabilistic Number Theory, Cambridge Univ. Press, Cambridge, 1995.

[15] A. Weingartner, Integers free of prime divisors from an interval II, preprint.

Department of Mathematics

Southern Utah University

Cedar City, UT 84720, U.S.A.

E-mail: weingartner@suu.edu

Received on 17.3.1999

and in revised form on 21.11.2000 